\title{
0 hemograma nas anemias microcíticas e hipocrômicas: aspectos diferenciais
}

\section{Blood tests in microcytic and hypochromic anemias: differential aspects}

Januária Fonseca Matos; Luci Maria Sant'Ana Dusse²; Karina Braga Gomes ${ }^{3}$; Rachel Versiani Bressane Stubert ${ }^{4}$; Mônica de F. Ribeiro Ferreira ${ }^{5}$; Roberta Coeli Neves Moreira ${ }^{6}$; Ana Paula Salles Moura Fernandes José Roberto de Faria8; Maria das Graças Carvalho

\section{unitermos}

Anemia microcítica

Talassemia

Anemia ferropriva

\section{resumo}

O diagnóstico diferencial das anemias microcíticas é clinicamente importante. Na tentativa de tornar esse diagnóstico menos oneroso e mais eficiente, o uso de parâmetros dos contadores automáticos tem sido sugerido. $\mathrm{O}$ objetivo deste estudo foi avaliar a eficiência diagnóstica de alguns parâmetros do hemograma na diferenciação das anemias microcíticas. Foram comparados os parâmetros hematológicos de 395 pacientes portadores de anemia ferropriva, anemia de doença crônica ou talassemia menor. O número de hemácias apresentou os maiores valores combinados de sensibilidade e especificidade na diferenciação dessas anemias. Em conclusão, a contagem de hemácias pode ser útil no diagnóstico diferencial de anemias microcíticas.

\section{abstract}

Differential diagnosis of microcytic anemia is clinically important. In an attempt to make this diagnosis more cost-effective, the use of some parameters obtained from automated blood count analyzers has been suggested. The objective of this study was to evaluate the efficiency of blood count parameters in differentiating microcytic anemias. Blood parameters were compared in 395 patients with iron deficiency anemia, chronic disease anemia or thalassemia minor. The number of red blood cells showed the highest combined sensitivity and specificity in differentiating these anemias. Hence, blood counts may be a useful tool in the differential diagnosis of microcytic anemias. key words

Microcytic anemia

Thalassemia

Iron deficiency anemia

\footnotetext{
1. Mestra em Ciências Farmacêuticas pela Universidade Federal de Minas Cerais (UFMG); professora do Instituto Federal de Minas Cerais - Campus Ouro Preto.

2. Doutora em Farmácia pela Universidade de São Paulo (USP); professora da Faculdade de Farmácia da UFMG.

3. Doutora em Ciências Farmacêuticas pela UFMG; professora da Faculdade de Farmácia da UFMG.

4. Farmacêutica bioquímica do Serviço de Patologia Clínica do Hospital Covernador Israel Pinheiro do Instituto de Previdência dos Servidores do Estado de Minas Cerais (IPSEMG)

5. Mestra em Ciências Farmacêuticas pela UFMC; farmacêutica bioquímica do Serviço de Patologia Clínica do Hospital Israel Pinheiro do IPSEMG.

6. Graduanda em Farmácia da UFMG; estagiária do Colégio Técnico da UFMG.

7. Doutora em Parasitologia pela UFMG; professora da Faculdade de Farmácia da UFMG.

8. Doutor em Medicina pela Universidade Federal de São Paulo (UNIFESP).

9. Doutora em Hematologia pela Universidade de Southampton; professora da Faculdade de Farmácia da UFMG.
} 


\section{Introdução}

O diagnóstico diferencial das anemias microcíticas e hipocrômicas (AMH) é clinicamente importante, uma vez que apresentam causas, tratamento e prognóstico diferentes. Atualmente, a comprovação diagnóstica dessas doenças é obtida pela realização de exames que avaliam o metabolismo do ferro, a eletroforese de hemoglobina e a dosagem de $\mathrm{HbA}_{2}$. Em determinadas condições de concomitância de doenças, como anemia ferropriva (AF) e anemia de doença crônica (ADC), os resultados dos exames padrão-ouro podem sofrer interferência da doença intercorrente, dificultando o diagnóstico.

Com o intuito de abreviar custos e aperfeiçoar o diagnóstico dessas anemias, vários parâmetros hematológicos têm sido sugeridos ${ }^{(4,13)}$. Entre estes, podem ser citados o volume corpuscular médio (VCM) e o número de hemácias, bem como outros da série vermelha. Além destes, o número de plaquetas também poderia auxiliar na distinção das anemias microcíticas, visto que muitas condições clínicas podem cursar com trombocitose reativa, entre elas AF e doenças inflamatórias ${ }^{(3,6)}$.

Este trabalho tem como objetivo discutir a eficiência de alguns parâmetros do tradicional hemograma no diagnóstico diferencial das $\mathrm{AMH}$.

\section{Material e métodos}

Foram coletadas amostras de 289 pacientes atendidos no Serviço de Patologia Clínica do Instituto de Previdência dos Servidores do Estado de Minas Gerais (IPSEMG), em Belo Horizonte, com idade acima de 18 anos, hemoglobina $<12 \mathrm{~g} / \mathrm{dl}$ (mulheres) e $13 \mathrm{~g} / \mathrm{dl}$ (homens) e VCM $<80 \mathrm{fl}$, caracterizando quadro de AMH. A avaliação hematológica foi realizada no contador Advia 120 (Bayer). Os níveis de ferritina foram determinados por quimioluminescência (Immulite e kits Immulite 2000 da DPC ${ }^{\circledR}$ ). Todos os pacientes apresentavam valores de ferritina abaixo dos valores de referência (28 a 397 ng/ml para homens e 6 a 159 ng/ml para mulheres), sendo classificados como portadores de anemia ferropriva.

Um segundo grupo de 53 pacientes foi recrutado no Serviço de Patologia Clínica do IPSEMG e no Hospital das Clínicas da Universidade Federal de Minas Gerais (UFMG). A avaliação hematológica foi realizada no contador Advia 120 (Bayer), para os pacientes selecionados no primeiro
Serviço, e no contador Sysmex XE2100 (Sysmex), para os indivíduos do Hospital. Ambos os contadores possuem controle de qualidade, permitindo inferir comparação dos dados. Esses pacientes também apresentavam idade acima 18 anos e $\mathrm{VCM}<80 \mathrm{fl}$, caracterizando quadro de microcitose e hipocromia (MH). Realizou-se eletroforese de hemoglobina utilizando fitas de acetato de celulose, em pH alcalino (8,6), em cuba/fonte Eletroforese Tecnow 7000. Em caso de suspeita de aumento de $\mathrm{HbA}_{2}$ fez-se a dosagem desta por eluição da fita eletroforética. $A$ ferritina foi dosada no aparelho Immulite ${ }^{\circledR}$ da $\mathrm{DPC}^{\circledR}$ em ambos os laboratórios. Os pacientes do segundo grupo com $\mathrm{MH}$ foram classificados como portadores de betatalassemia menor, quando apresentaram níveis séricos de ferritina normais, porém com $\mathrm{HbA}_{2}$ aumentada $(>3,5 \mathrm{~g} / \mathrm{dl}$ ). Por outro lado, os pacientes com $\mathrm{MH}$, com perfil hematológico sugestivo de alfatalassemia, embora com pesquisa de $\mathrm{HbH}$ negativa, foram confirmados como portadores do distúrbio alfatalassêmico, na forma menor, por biologia molecular. Os cinco pacientes que apresentaram esse quadro tiveram mutação 3.7.

Um terceiro grupo, também recrutado no Serviço de Patologia do IPSEMG, foi composto de 53 pacientes com AMH, cujos níveis de ferritina estavam normais ou aumentados e, juntamente com dados clínicos, caracterizavam ADC.

$\mathrm{Na}$ tentativa de instituir os valores supostamente úteis na diferenciação das $\mathrm{AMH}$, foi plotada a curva receiver operating characteristics (ROC), utilizando o software Prisma 5, que estabelecia os pontos de corte (cutoffs) para cada um dos parâmetros hematológicos e, ainda, determinava a sensibilidade e a especificidade para cada um desses pontos (Tabela).

\section{Resultados}

Foi possível observar, pela análise da curva ROC, que a contagem de hemácias constitui o principal parâmetro auxiliar na discriminação das AMH. A Tabela apresentou os maiores valores de sensibilidade e especificidade nos pontos de corte para a contagem de hemácias.

\section{Discussão}

Uma análise dos dados referentes aos parâmetros hematológicos demonstrados na Tabela, os quais visavam à discriminação entre as $\mathrm{AMH}$, mostrou que a contagem de 


\section{Sensibilidade, especificidade e pontos de corte (cutoffs) de parâmetros hematológicos na}

Tabela diferenciação entre AF, ADC e talassemia

\begin{tabular}{|c|c|c|c|c|c|c|c|c|c|}
\hline \multirow[b]{2}{*}{ Parâmetro } & \multicolumn{3}{|c|}{ AF x talassemia } & \multicolumn{3}{|c|}{$A F \times A D C$} & \multicolumn{3}{|c|}{ Talassemia $x$ ADC } \\
\hline & Cutoffe & Sensibilidade & Especificidade & Cutoff & Sensibilidade & Especificidade & Cutoff & Sensibilidade & Especificidade \\
\hline $\mathrm{Hm}\left(10^{6} / \mu l\right)$ & $>5$ & $\begin{array}{c}83 \\
(70,2 ; 91,9)\end{array}$ & $\begin{array}{c}85,1 \\
(80,5 ; 89,0)\end{array}$ & $>4$ & $\begin{array}{c}75,5 \\
(61,7 ; 86,2)\end{array}$ & $\begin{array}{c}85,5 \\
(80,9 ; 89,3)\end{array}$ & $<4,4$ & $\begin{array}{c}90,6 \\
(79,3 ; 96,9)\end{array}$ & $\begin{array}{c}92,4 \\
(81,8 ; 97,9)\end{array}$ \\
\hline $\mathrm{Hb}(\mathrm{g} / \mathrm{dl})$ & $>10,7$ & $\begin{array}{c}71,7 \\
(57,6 ; 83,2)\end{array}$ & $\begin{array}{c}75,8 \\
(70,4 ; 80,6)\end{array}$ & $>9,8$ & $\begin{array}{c}62,3 \\
(47,9 ; 75,2)\end{array}$ & $\begin{array}{c}59,9 \\
(54 ; 65,6)\end{array}$ & $<10,4$ & $\begin{array}{c}81,1 \\
(68 ; 90,6)\end{array}$ & $\begin{array}{c}81,1 \\
(68 ; 90,6)\end{array}$ \\
\hline VCM (fl) & $<70,2$ & $\begin{array}{c}83 \\
(70,2 ; 91,9)\end{array}$ & $\begin{array}{c}63 \\
(57,1 ; 68,6)\end{array}$ & $<75,2$ & $\begin{array}{c}79,2 \\
(65,9 ; 89,2)\end{array}$ & $\begin{array}{c}74,4 \\
(69 ; 79,3)\end{array}$ & $>71,3$ & $\begin{array}{c}90,6 \\
(79,3 ; 96,9)\end{array}$ & $\begin{array}{c}88,7 \\
(77 ; 95,7)\end{array}$ \\
\hline HCM (pg) & $<21,7$ & $\begin{array}{c}73,6 \\
(59,7 ; 84,7)\end{array}$ & $\begin{array}{c}62,3 \\
(56,4 ; 67,9)\end{array}$ & $<24,1$ & $\begin{array}{c}77,4 \\
(63,8 ; 87,7)\end{array}$ & $\begin{array}{c}77,2 \\
(71,9 ; 81,9)\end{array}$ & $>23$ & $\begin{array}{c}86,8 \\
(74,7 ; 94,5)\end{array}$ & $\begin{array}{c}86,8 \\
(74,7 ; 94,5)\end{array}$ \\
\hline CHCM (g/dl) & $>31,3$ & $\begin{array}{c}73,6 \\
(59,7 ; 84,7)\end{array}$ & $\begin{array}{c}52,2 \\
(46,3 ; 58,1)\end{array}$ & $<32,1$ & $\begin{array}{c}71,7 \\
(57,6 ; 83,2)\end{array}$ & $\begin{array}{c}75,4 \\
(70 ; 80,3)\end{array}$ & $>32,8$ & $\begin{array}{c}86,8 \\
(74,7 ; 94,5)\end{array}$ & $\begin{array}{c}49,1 \\
(35,1 ; 63,2)\end{array}$ \\
\hline $\mathrm{Pla}\left(10^{3} / \mu \mathrm{l}\right)$ & $<271,5$ & $\begin{array}{c}73,6 \\
(59,7 ; 84,7)\end{array}$ & $\begin{array}{c}71,3 \\
(65,7 ; 76,4)\end{array}$ & - & & & $>265,5$ & $\begin{array}{c}60,4 \\
(46,0 ; 73,5)\end{array}$ & $\begin{array}{c}66 \\
(51,7 ; 78,5)\end{array}$ \\
\hline
\end{tabular}

a, b e c: valores de Cutoff correspondentes a talassemia menor, anemia ferropriva e anemia de doença crônica, respectivamente.

AF: anemia ferropriva; ADC: anemia de doença crônica; Hm: número de hemácias; Hb: hemoglobina; VCM: volume corpuscular médio; HCM: hemoglobina corpuscular média, CHCM: concentração de hemoglobina corpuscular média; Pla: número de plaquetas.

hemácias se destacou por apresentar os maiores valores combinados de sensibilidade e especificidade e, portanto, maior área sob a curva ROC, em relação aos outros parâmetros avaliados.

Os dados analisados conjuntamente mostram que um cutoff superior a cinco milhões é compatível com talassemia menor, sendo importante na discriminação entre esse distúrbio e anemia ferropriva, enquanto na discriminação entre AF e ADC, o valor de cutoff foi de quatro milhões; acima deste, o paciente é portador, possivelmente, de anemia ferropriva. No caso de comparação entre ADC e talassemia, o valor de cutoff foi de 4,4 milhões, interpretando-se, assim, que um paciente com contagem de hemácias inferior a 4,4 milhões é um possível portador de ADC. Esses diferentes valores de cutoff estão condizentes com a fisiopatologia dos distúrbios.

O número de hemácias apresenta os menores valores no grupo $A D C$, refletindo, provavelmente, a fisiopatologia do distúrbio, no qual se verifica inibição da proliferação e da diferenciação dos progenitores eritroides pela ação de citocinas pró-inflamatórias ${ }^{(7)}$. Por outro lado, o maior número de eritrócitos ocorre na talassemia menor. Esse achado se relaciona com a fisiopatologia desse distúrbio, em que a precipitação das cadeias globínicas, em excesso nos precursores eritroides e eritrócitos circulantes, resulta em discreta eritropoese ineficaz, aumento da eritropoetina e, finalmente, leve a moderada expansão da eritropoese pela medula óssea na tentativa de compensar a anemia. Esse mecanismo se manifesta por um aumento no número de hemácias do sangue ${ }^{(8,9,12)}$. De fato, o número de hemácias é útil como parâmetro diagnóstico auxiliar, uma vez que as talassemias menores produzem anemia microcítica associada à eritrocitose ${ }^{(2)}$.

Segundo Hilliard e Berkow ${ }^{(9)}$, os pacientes com betatalassemia menor apresentam maior número de hemácias para o grau de anemia quando comparados com os pacientes portadores de anemia ferropriva. Já em pacientes portadores de AF, observam-se valores intermediários para o número de hemácias, sem maiores decréscimos. Isso ocorre porque na anemia ferropriva a proliferação de progenitores eritroides não se encontra comprometida como na ADC. Realmente, o mecanismo desencadeador da anemia ferropriva se refere à restrição de ferro à medula óssea, comprometendo de forma mais importante a síntese da hemoglobina e não a produção dos eritrócitos. Outras causas de anemia microcítica, como a ADC, estão tipicamente relacionadas com diminuição do número de hemácias proporcional ao decréscimo da concentração de hemoglobina ${ }^{(2)}$.

Vários trabalhos investigaram a importância da contagem de eritrócitos na diferenciação das anemias microcíticas e hipocrômicas ${ }^{(1,4,5,11,10)}$, verificando que eritrocitose foi comumente observada nos pacientes com talassemia menor e distintamente incomum nos indivíduos portadores de anemia ferropriva. A contagem de eritrócitos acima de cinco 
milhões também é um índice bastante útil para a triagem das referidas anemias e para a suspeita de betatalassemia menor ${ }^{(1,4,5,10,11)}$.

Em conclusão, o presente estudo sugere que, mesmo não sendo possível alcançar um diagnóstico definitivo das anemias microcíticas e hipocrômicas baseando-se apenas em parâmetros obtidos dos contadores automáticos, esses simples cálculos podem ser potencialmente úteis no rastreamento de pacientes portadores desse tipo de anemia. Apesar de sob o ponto de vista individual não ser possível afirmar categoricamente o diagnóstico correto para cada paciente, uma vez que há considerável sobreposição de valores dos parâmetros hematológicos, a análise realizada por meio da curva ROC reforça a ideia de que a contagem de hemácias constitui o principal parâmetro auxiliar na discriminação das anemias microcíticas. Dessa forma, uma contagem exata do número de hemácias poderia oferecer um direcionamento para a escolha de testes laboratoriais confirmatórios, com custos mais altos, se necessário, e evitar um tratamento inapropriado, resultando, assim, em significante redução de gastos pelo sistema de saúde.

\section{Referências}

1. BEYAN, C.; KAPTAN, K.; IFRAN, A. Predictive value of discrimination indices in differential diagnosis of iron deficiency anemia and beta-thalassemia trait. Eur J Haematol, v. 78, n. 6, p. 5246, 2007.

2. CLARKE, G. M.; HIGGINS, T. N. Laboratory investigation of hemoglobinopathies and thalassemias: review and update. Clin Chem, v. 46, n. 8, p. 1284-90, 2000.

3. DAN, K. Thrombocytosis in iron deficiency anemia. Intern Med, v. 44, n. 10, p. 1025-6, 2005.

4. DEMIR, A. et al. Most reliable indices in differentiation between thalassemia trait and iron deficiency anemia. Pediatr Int, v. 44, n. 6, p. 612-6, 2002.

5. FLYNN, M. M.; REPPUN, T. S.; BHAGAVAN, N. V. Limitations of red blood cell distribution width (RDW) in evaluation of microcytosis. Am J Clin Pathol, v. 85, n. 4, p. 445-9, 1986.

6. GILES, C. The platelet count and mean platelet volume. Br J Haematol, v. 48, n. 1, p. 317, 1981.

7. GUIDI, G. C.; SANTONASTASO, C. L. Advancements in anemias related to chronic conditions. Clin Chem Lab Med, v. 48, n. 9, p. 1217-26, 2010.
8. HANDIN, R. J.; LUX, S. E.; STOSSEL, T. P. Blood: principles and practice of hematology. 2. ed. Philadelphia: Lippincott Williams \& Wilkins, 2003, 2304 p.

9. HILLIARD, L. M.; BERKOW, R. L. The thalassemia syndromes. Prim Care Update for Ob/Gyns, v. 3, n. 5, p. 157-62, 1996.

10. MADAN, N. et al. Phenotypic expression of hemoglobin A2 in beta-thalassemia trait with iron deficiency. Ann Hematol, v. 77, n. 3, p. 936, 1998.

11. MELO, M. R. et al. Uso de índices hematimétricos no diagnóstico diferencial de anemias microcíticas: uma abordagem a ser adotada? Rev Assoc Med Bras, v. 48, n. 3, p. 222-4, 2002.

12. SCHRIER, S. L.; ANGELUCCI, E. New strategies in the treatment of the thalassemias. Annu Rev Med, v. 56, p. 157-71, 2005.

13. WENTWORTH, P.; WENTWORTH, S. The value of the MCV index in the diagnosis of thalassemic and nonthalassemic microcytosis. Am J Clin Pathol, v. 107, n. 5, p. 619, 1997.
Endereço para correspondência

Maria das Graças Carvalho

Laboratório de Hematologia Departamento de Análises Clínicas e Toxicológicas Faculdade de Farmácia da Universidade Federal de Minas Cerais

Av. Antônio Carlos, 6627 - Pampulha

CEP: $31270-010$ - Belo Horizonte-MG

Tel.: (31) 3409-6881

Fax: (31) 3409-6895

e-mail: mgcarvalho@farmacia.ufmg.br 